\title{
GENERALIZED DIMENSION DISTORTION UNDER MAPPINGS OF SUB-EXPONENTIALLY INTEGRABLE DISTORTION
}

\section{Tapio Rajala, Aleksandra Zapadinskaya and Thomas Zürcher}

\author{
University of Jyväskylä, Department of Mathematics and Statistics \\ P.O. Box 35, FI-40014 University of Jyväskylä, Finland; tapio.m.rajala@jyu.fi \\ University of Jyväskylä, Department of Mathematics and Statistics
}

P.O. Box 35, FI-40014 University of Jyväskylä, Finland; aleksandra.zapadinskaya@jyu.fi

University of Jyväskylä, Department of Mathematics and Statistics

P.O. Box 35, FI-40014 University of Jyväskylä, Finland; thomas.t.zurcher@jyu.fi

\begin{abstract}
We prove a dimension distortion estimate for mappings of sub-exponentially integrable distortion in Euclidean spaces, which is sharp modulo a constant.
\end{abstract}

\section{Introduction}

The roots of our studies lie in [7], where the following was proved: given a planar $K$-quasiconformal mapping $f$ and a set $E$ with $\operatorname{dim}_{\mathscr{H}} E<2$, we have $\operatorname{dim}_{\mathscr{H}} f(E) \leq$ $\beta<2$, where $\beta$ depends only on $K$ and the Hausdorff $\operatorname{dimension} \operatorname{dim}_{\mathscr{H}} E$ of the set $E$. Later, it was shown that the same is true in higher dimensions with $\beta$ depending on the dimension of the underlying space as well as on $K$ and on $\operatorname{dim}_{\mathscr{H}} E$ (see [6]). These results rely on the higher integrability of the Jacobian of a quasiconformal mapping $[4,6]$.

Recent extensions take a wider class of mappings into consideration. A continuous mapping $f \in W_{\text {loc }}^{1,1}\left(\Omega ; \mathbf{R}^{n}\right)\left(\Omega \subset \mathbf{R}^{n}\right.$ is a domain) is called a mapping of finite distortion, if its Jacobian $J_{f}$ is locally integrable and there exists a measurable function $K: \Omega \rightarrow[1, \infty[$ such that

$$
|D f(x)|^{n} \leq K(x) J_{f}(x)
$$

for almost every $x \in \Omega$. We denote the optimal distortion function of $f$ by $K_{f}$ :

$$
K_{f}(x)= \begin{cases}\frac{|D f(x)|^{n}}{J_{f}(x)}, & J_{f}(x) \neq 0, \\ 1, & \text { otherwise. }\end{cases}
$$

An assumption on $K_{f}$ that still guarantees some of the properties of quasiconformal mappings is the so-called exponential integrability. This condition requires that $\exp \left(\lambda K_{f}\right)$ is locally integrable for some $\lambda>0$. In this case, $f$ is called a mapping of $\lambda$-exponentially integrable distortion.

doi:10.5186/aasfm.2011.3631

2010 Mathematics Subject Classification: Primary 30C62.

Key words: Mappings of finite distortion, sub-exponential distortion, generalized Hausdorff measure, Hausdorff dimension.

The second author was partially supported by the Academy of Finland, grant no. 120972, and the third author was supported by the Swiss National Science Foundation. 
Such mappings satisfy Lusin's condition N, i.e. they map sets of measure zero to sets of measure zero, [14]. However, in [12, Proposition 5.1], a mapping $f: \mathbf{R}^{n} \rightarrow \mathbf{R}^{n}$ of finite exponentially integrable distortion that maps sets of Hausdorff dimension less than $n$ to sets of Hausdorff dimension $n$ was constructed.

Still it was possible to obtain reasonable dimension distortion results in terms of generalized Hausdorff measure (see the next section for the definition). In [12], it was shown that there exists a constant $k_{n}$, depending only on $n$, such that if $f: \mathbf{R}^{n} \rightarrow \mathbf{R}^{n}$ is a homeomorphism with $\lambda$-exponentially integrable distortion for some $\lambda$, then $\mathscr{H}^{h}\left(f\left(S^{n-1}\right)\right)<\infty$ for all $p<k_{n} \lambda$, where $\mathscr{H}^{h}$ is the generalized Hausdorff measure with gauge function $h(t)=t^{n} \log ^{p}(1 / t)$.

This result was improved for the planar case in [19], where the circle $S^{1}$ was replaced by a general set $E$ of Hausdorff dimension less than two: we have $\mathscr{H}^{h}(f(E))=$ 0 for all $p<\lambda$, where $h(t)=t^{2} \log ^{p}(1 / t)$, if $f$ is a mapping of $\lambda$-exponentially integrable distortion. The proof is based on the higher regularity for the weak derivatives of the mapping $f[1]$ and dimension distortion estimates for Orlicz-Sobolev mappings. See $[18,21]$ for related results in the plane and [22] for the generalization to higher dimensions.

The assumption of exponential integrability for the distortion is further relaxed by replacing it with a more general Orlicz condition. That is, given a mapping of finite distortion $f: \Omega \rightarrow \mathbf{R}^{n}$, one may assume $e^{\mathscr{A}\left(K_{f}\right)} \in L_{\text {loc }}^{1}(\Omega)$, where $\mathscr{A}:[1, \infty[\rightarrow[0, \infty[$ is a smooth increasing function such that (see [2, Section 20.5])

$$
\int_{1}^{\infty} \frac{\mathscr{A}(t)}{t^{2}} d t=\infty .
$$

In particular, when $\mathscr{A}(t)=p \frac{t}{1+\log t}-p$, for some $p>0$, the mapping $f$ is called a mapping of sub-exponentially integrable distortion. Dimension distortion in this particular case is examined in this paper.

Let us agree that from now on, $\Omega$ is always an open set in $\mathbf{R}^{n}, n \geq 2$. Denote $h_{n, \beta}(t)=t^{n}(\log \log (1 / t))^{\beta}$. We have the following theorem.

Theorem 1. There exists a constant $c>0$, which depends only on the dimension $n$ of the underlying space, such that for every homeomorphism of finite distortion $f \in W_{\mathrm{loc}}^{1,1}\left(\Omega ; \mathbf{R}^{n}\right), \Omega \subset \mathbf{R}^{n}$, with

$$
e^{\frac{K_{f}}{1+\log K_{f}}} \in L_{\mathrm{loc}}^{p}(\Omega)
$$

for some $p>0$, we have $\mathscr{H}^{h_{n, \beta}}(f(E))=0$ for all $\beta<c p$, whenever $E \subset \Omega$ is such that $\operatorname{dim}_{\mathscr{H}} E<n$.

When $n=2$, the assumption on $f$ to be a homeomorphism is not necessary due to Stoilow factorization (see Section 5 for the details). The constant $c$ equals one in this case:

Theorem 2. Let $f \in W_{\text {loc }}^{1,1}\left(\Omega ; \mathbf{R}^{2}\right), \Omega \subset \mathbf{R}^{2}$, be a mapping of finite distortion with

$$
e^{\frac{K_{f}}{1+\log K_{f}}} \in L_{\mathrm{loc}}^{p}(\Omega)
$$

for some $p>0$. Then $\mathscr{H}^{h_{2, \beta}}(f(E))=0$ for all $\beta<p$, whenever $E \subset \Omega$ is such that $\operatorname{dim}_{\mathscr{H}} E<2$. 
The following example shows that Theorems 1 and 2 are sharp modulo a constant.

Example 1. There exists a constant $C \geq 1$ depending only on $n$, such that for any $\beta>0$ and $\varepsilon \in] 0, \beta\left[\right.$, we may construct sets $\mathscr{C}, \mathscr{C}^{\prime} \subset[0,1]^{n}$, satisfying $\operatorname{dim}_{\mathscr{H}} \mathscr{C}<$ $n$ and $\mathscr{H}^{h_{n, \beta}}\left(\mathscr{C}^{\prime}\right)>0$, and a mapping of finite distortion $f \in W^{1,1}\left([0,1]^{n} ; \mathbf{R}^{n}\right)$, such that

and $f(\mathscr{C})=\mathscr{C}^{\prime}$.

$$
e^{\frac{K_{f}}{1+\log K_{f}}} \in L^{\frac{1}{C} \beta-\varepsilon}\left([0,1]^{n}\right)
$$

The main auxiliary result, used in the proof of the theorems, is higher integrability for the Jacobian of a mapping of sub-exponentially integrable distortion, proved in [5] for general dimensions and refined in [8], where a sharp estimate for the higher integrability of the Jacobian of a planar mapping was obtained. Those estimates are combined with the methods used in $[18,21]$ for the case of exponentially integrable distortion.

One could extend the results presented here to a case of a more general function $\mathscr{A}$, in particular, when $\mathscr{A}$ is given by

$$
\mathscr{A}_{p, k}(t)=\frac{p t}{1+\log (t) \log (\log (e-1+t)) \cdots \log \left(\ldots\left(\log \left(e^{e^{. \cdot e}}-1+t\right)\right) \ldots\right)}-p,
$$

where $k$ means that the last logarithmic expression is a $k$-th iterated logarithm (a case studied in [8, Theorem 4]). However, we leave the results in the presented form, because the construction demonstrating sharpness is quite complicated even in the case of a single logarithm.

Let us remark that the integrability assumption in (1) is essential if one wishes to obtain dimension distortion estimates for mappings of finite distortion. Indeed, Section 5 of [14] provides a construction of a homeomorphism $f$ of finite distortion $K$ with $e^{\mathscr{A}(K)} \in L_{\text {loc }}^{1}$ for some function $\mathscr{A}:[1, \infty[\rightarrow[0, \infty[$ such that

$$
\int_{1}^{\infty} \frac{\mathscr{A}(t)}{t^{2}} d t<\infty
$$

and $f$ maps a set of Hausdorff dimension strictly less than the dimension $n$ of the underlying space to a set of positive Lebesgue measure. More precisely, $\mathscr{A}$ is taken as $\mathscr{A}(t)=p \frac{t}{\log ^{2}(e+t)}-p$ for some particular $p>0$. See [16] for refined constructions.

\section{Definitions}

Let us agree on some notation. For a set $V \subset \mathbf{R}^{n}$ and a number $\delta>0, V+\delta$ denotes the set $\left\{y \in \mathbf{R}^{n}: \operatorname{dist}(y, V)<\delta\right\}$.

Always when we introduce a constant using the notation $C=C(\cdot)$, we mean that the constant $C$ depends only on the parameters listed in the parentheses.

We write $\mathscr{H}^{h}(A)$ for the generalized Hausdorff measure of a set $A$, given by

$$
\mathscr{H}^{h}(A)=\lim _{\delta \rightarrow 0} \mathscr{H}_{\delta}^{h}(A)
$$

where

$$
\mathscr{H}_{\delta}^{h}(A)=\inf \left\{\sum_{i=1}^{\infty} h\left(\operatorname{diam} U_{i}\right): A \subset \bigcup_{i=1}^{\infty} U_{i}, \operatorname{diam} U_{i} \leq \delta\right\}
$$


and $h$ is a dimension gauge (a non-decreasing function with $\lim _{t \rightarrow 0+} h(t)=h(0)=0$ ). If $h(t)=t^{\alpha}$ for some $\alpha \geq 0$, we simply put $\mathscr{H}^{\alpha}$ for $\mathscr{H}^{t^{\alpha}}$ and call it the Hausdorff $\alpha$-dimensional measure, and the Hausdorff dimension $\operatorname{dim}_{\mathscr{H}} A$ of the set $A$ is the smallest $\alpha_{0} \geq 0$ such that $\mathscr{H}^{\alpha}(A)=0$ for any $\alpha>\alpha_{0}$.

Let us recall the definition of Orlicz classes. An Orlicz function is a continuous increasing function $P:\left[0, \infty\left[\rightarrow\left[0, \infty\left[\right.\right.\right.\right.$ such that $P(0)=0$ and $\lim _{t \rightarrow \infty} P(t)=\infty$. Given an Orlicz function $P$, we denote by $L^{P}(\Omega)$ the Orlicz class of integrable functions $h: \Omega \rightarrow \mathbf{R}$ such that

$$
\int_{\Omega} P(\nu|h|)<\infty
$$

for some $\nu=\nu(h)>0$. An Orlicz-Sobolev class $W^{1, P}(\Omega)$ is a class of mappings $g \in W^{1,1}\left(\Omega ; \mathbf{R}^{2}\right)$ such that all the distributional partial derivatives of $g$ are in the class $L^{P}(\Omega)$.

Finally, given a mapping $f \in W_{\text {loc }}^{1,1}\left(\Omega, \mathbf{R}^{n}\right)$, we write the equality $\operatorname{Det} D f=J_{f}$, if the distributional determinant Det $D f[3]$ coincides with the pointwise Jacobian $J_{f}$, that is, if

$$
\int_{\Omega} f_{1}(x) J_{\tilde{f}}(x) d x=-\int_{\Omega} \varphi(x) J_{f}(x) d x
$$

holds for each $\varphi \in C_{0}^{\infty}(\Omega)$ (here $f=\left(f_{1}, \ldots, f_{n}\right)$ and $\tilde{f}=\left(\varphi, f_{2}, \ldots, f_{n}\right)$ ). See [13, $9,10,20]$ for some conditions on the regularity of the weak derivatives of $f$ sufficient to guarantee this equality.

\section{Example}

Fix $\beta>0$. Let us construct the mapping in Example 1. We start by defining the pre-image and image Cantor sets $\mathscr{C}$ and $\mathscr{C}^{\prime}$, respectively. Fix $\left.\sigma \in\right] 0,1 / 2[$. The set $\mathscr{C}$ is obtained as a Cartesian product $\mathscr{C}_{1} \times \ldots \times \mathscr{C}_{1}$ ( $n$ times $)$, where $\mathscr{C}_{1}$ is a Cantor set on the real line. In order to construct $\mathscr{C}_{1}$, take a unit segment $I=[0,1]$ and divide it into eight equal parts. Consider eight closed intervals $I_{j}^{3}, j=1, \ldots, 8$, of length $\sigma^{3}$, each taken in the middle of one of the obtained segments. At the further steps, the intervals considered are always divided into two parts. Given $2^{k}, k \geq 3$, intervals $I_{j}^{k}, j=1, \ldots, 2^{k}$, of length $\sigma^{k}$, we divide each of them into two parts and take $2^{k+1}$ closed intervals $I_{j}^{k+1}, j=1, \ldots, 2^{k+1}$, of length $\sigma^{k+1}$, each in the middle of one of the obtained parts. Finally, $\mathscr{C}_{1}$ is taken as $\bigcap_{k \geq 3} \bigcup_{j=1}^{2^{k}} I_{j}^{k}$. The Hausdorff measure $\mathscr{H}^{\alpha}\left(\mathscr{C}_{1}\right)$ of the set $\mathscr{C}_{1}$ for $\left.\alpha \in\right] \frac{\log 2}{\log (1 / \sigma)}, 1[$ may be estimated as

$$
\mathscr{H}^{\alpha}\left(\mathscr{C}_{1}\right) \leq \inf _{k \geq 3}\left\{2^{k} \sigma^{\alpha k}\right\}=0,
$$

so, $\operatorname{dim}_{\mathscr{H}} \mathscr{C}_{1}<1$, and thus, $\operatorname{dim}_{\mathscr{H}}(\underbrace{\mathscr{C}_{1} \times \ldots \times \mathscr{C}_{1}}_{n \text { times }})<n$.

The image set $\mathscr{C}^{\prime}$ is constructed similarly, but at the $k$-th step, $k \geq 3$, the length of the intervals chosen is $l_{k}=2^{-k} \log ^{-\beta / n} k$ instead of $\sigma^{k}$. For any $k \geq 3$, the set $\mathscr{C}^{\prime}$ can be covered by $2^{n k}$ cubes of side length $l_{k}$. Let us see that $\mathscr{H}^{h_{n, \beta}}\left(\mathscr{C}^{\prime}\right)>0$. We prove it using the mass distribution principle. We have

$$
\lim _{k \rightarrow \infty} 2^{n k} h_{n, \beta}\left(l_{k}\right)=\lim _{k \rightarrow \infty} 2^{n k} l_{k}^{n}\left(\log \log \left(1 / l_{k}\right)\right)^{\beta}=1 .
$$


Put $m:=\inf _{k \geq 3}\left\{2^{n k} h_{n, \beta}\left(l_{k}\right)\right\}>0$ and let $\mu$ be the uniformly distributed probability measure supported by $\mathscr{C}^{\prime}$. Suppose also that $\delta>0$ is so small that $h_{n, \beta}(t)$ is increasing in $t$ on the interval ] $0, \delta\left[\right.$. Then for any $U \subset \mathbf{R}^{n} \operatorname{such}$ that $l_{k+1} \leq \operatorname{diam} U<\min \left\{\delta, l_{k}\right\}$ for some $k \geq 3$, we have

$$
\mu(U) \leq 2^{n} \cdot 2^{-n k} \leq \frac{2^{2 n} h_{n, \beta}\left(l_{k+1}\right)}{m} \leq \frac{2^{2 n} h_{n, \beta}(\operatorname{diam} U)}{m} .
$$

Thus, for any covering $\bigcup_{i} U_{i}$ of the set $\mathscr{C}^{\prime}$, such that $\operatorname{diam} U_{i}<\min \left\{\delta, l_{3}\right\}, i=1,2, \ldots$, we observe

$$
\sum_{i=1}^{\infty} h_{n, \beta}\left(\operatorname{diam} U_{i}\right) \geq \frac{m}{2^{2 n}} \sum_{i=1}^{\infty} \mu\left(U_{i}\right) \geq \frac{m}{2^{2 n}} \mu\left(\bigcup_{i=1}^{\infty} U_{i}\right)=\frac{m}{2^{2 n}}>0 .
$$

Hence $\mathscr{H}_{\delta_{1}, \beta}^{h_{n, \beta}}\left(\mathscr{C}^{\prime}\right) \geq m / 2^{2 n}>0$ for all $\delta_{1} \leq \min \left\{\delta, l_{3}\right\}$, therefore $\mathscr{H}^{h_{n, \beta}}\left(\mathscr{C}^{\prime}\right)>0$.

Let us denote by $Q_{k, j}$ with $k=3,4, \ldots$ and $j=1, \ldots, 2^{n k}$ the cubes of the side length $\sigma^{k}$, appearing on the pre-image side at the $k$-th step of the construction. Write $q_{k, j}$ for the centres of these cubes. Next, let $A_{k, j}$ for $k=3,4, \ldots$ and $j=1, \ldots, 2^{n k}$ denote the frames

$$
\left\{x \in \mathbf{R}^{n}: r_{k}<\left|x-q_{k, j}\right|_{\infty}<R_{k}\right\},
$$

where $r_{k}=\sigma^{k} / 2$ for $k \geq 3, R_{k}=\sigma^{k-1} / 4$ for $k \geq 4, R_{3}=1 / 16$ and $|\cdot|_{\infty}$ is the maximum norm:

$$
|x|_{\infty}=\max \left\{\left|x_{i}\right|\right\}_{i=1}^{n} .
$$

The inner boundary $\left\{x \in \mathbf{R}^{n}:\left|x-q_{k, j}\right|_{\infty}=r_{k}\right\}$ of the frame $A_{k, j}$ is exactly the boundary of the cube $Q_{k, j}$. Let us introduce similar notation for the image side. Write $Q_{k, j}^{\prime}$ with $k=3,4, \ldots$ and $j=1, \ldots, 2^{n k}$ for the cubes with the side length $l_{k}=2^{-k} \log ^{-\beta / n} k$ and $q_{k, j}^{\prime}$ for the centres of these cubes. Finally, $A_{k, j}^{\prime}$ for $k=3,4, \ldots$ and $j=1, \ldots, 2^{n k}$ denote the frames

$$
\left\{x \in \mathbf{R}^{n}: r_{k}^{\prime}<\left|x-q_{k, j}^{\prime}\right|_{\infty}<R_{k}^{\prime}\right\},
$$

where $r_{k}^{\prime}=2^{-k-1} \log ^{-\beta / n} k$ for $k \geq 3, R_{k}^{\prime}=2^{-k-1} \log ^{-\beta / n}(k-1)$ for $k \geq 4$ and $R_{3}^{\prime}=1 / 16$.

We are ready to construct a mapping $f:[0,1]^{n} \rightarrow \mathbf{R}^{n}$ such that $f(\mathscr{C})=\mathscr{C}^{\prime}$. The construction is similar to the one in [12, Proposition 5.1]. First, let

$$
a_{k}=\frac{R_{k}^{\prime}-r_{k}^{\prime}}{R_{k}-r_{k}} \quad \text { and } \quad b_{k}=\frac{R_{k} r_{k}^{\prime}-R_{k}^{\prime} r_{k}}{R_{k}-r_{k}}
$$

for $k \geq 3$. Then, define $f_{3}$ as

$$
f_{3}(x)= \begin{cases}\left(a_{3}\left|x-q_{3, j}\right|_{\infty}+b_{3}\right) \frac{x-q_{3, j}}{\left|x-q_{3, j}\right|_{\infty}}+q_{3, j}^{\prime}, & x \in \bar{A}_{3, j}, j=1, \ldots, 8^{n}, \\ \frac{r_{3}^{\prime}}{r_{3}}\left(x-q_{3, j}\right)+q_{3, j}^{\prime}, & x \in Q_{3, j}, j=1, \ldots, 8^{n} .\end{cases}
$$

We proceed by putting

$$
f_{k}(x)= \begin{cases}\left(a_{k}\left|x-q_{k, j}\right|_{\infty}+b_{k}\right) \frac{x-q_{k, j}}{\left|x-q_{k, j}\right|_{\infty}}+q_{k, j}^{\prime}, & x \in A_{k, j}, j=1, \ldots, 2^{n k}, \\ \frac{r_{k}^{\prime}}{r_{k}}\left(x-q_{k, j}\right)+q_{k, j}^{\prime}, & x \in \bar{Q}_{k, j}, j=1, \ldots, 2^{n k}, \\ f_{k-1}(x), & \text { otherwise, }\end{cases}
$$

for $k>3$. The mapping $f$ is obtained as the pointwise limit $f=\lim _{k \rightarrow \infty} f_{k}$.

It is a Sobolev mapping. Indeed, let us first see that it is ACL (absolutely continuous on lines). Take a line on the pre-image side parallel to the $x_{1}$-axis that 
does not hit the initial Cantor set $\mathscr{C}$. On this line, the mapping $f$ coincides with one of the mappings $f_{k_{0}}$ in our sequence, which is Lipschitz and, therefore, absolutely continuous along the considered line. Since $\mathscr{C}_{1}$ has vanishing Lebesgue measure $\mathscr{L}^{1}$, it follows that $f$ is ACL. Next, let us check the integrability of the differential of $f$. Its behaviour is essentially defined by the behaviour of $f$ on the cubical collars $A_{k, j}$, where it is given by

$$
\left(a_{k}|x|_{\infty}+b_{k}\right) \frac{x}{|x|_{\infty}}, \quad r_{k}<|x|_{\infty}<R_{k}
$$

up to a translation. By Lemma 4.1 in [15], there exists a constant $C_{0}=C_{0}(n) \geq 1$ such that

$$
|D f(x)|=\left|D f_{k}(x)\right| \leq C_{0} \max \left\{a_{k}, a_{k}+\frac{b_{k}}{\left|x-q_{k, j}\right|_{\infty}}\right\} \text { for a.e. } x \in A_{k, j} .
$$

It is possible to find $k_{0} \in \mathbf{N}$ such that $b_{k}>0$ for all $k \geq k_{0}$. Then we have

$$
|D f(x)| \leq C_{0}\left(a_{k}+\frac{b_{k}}{\left|x-q_{k, j}\right|_{\infty}}\right) \leq C_{0} \frac{r_{k}^{\prime}}{r_{k}}
$$

for almost every $x \in A_{k, j}$, when $k \geq k_{0}$. So, the integrability of the differential of $f$ may be estimated with help of the following series:

$$
\int_{[0,1]^{n}}|D f| \leq C_{1}+C_{0} \sum_{k=k_{0}}^{\infty}(2 \sigma)^{n(k-1)} \frac{2^{-k} \log ^{-\beta / n} k}{\sigma^{k}}=C_{1}+C_{2} \sum_{k=k_{0}}^{\infty}(2 \sigma)^{(n-1) k} \log ^{-\beta / n} k,
$$

where $C_{1}=C_{1}(n, \sigma, \beta)$ and $C_{2}=C_{2}(n, \sigma)$ are positive constants. This series converges by the Ratio Test, since

$$
\lim _{k \rightarrow \infty} \frac{\log ^{-\beta / n}(k+1)}{\log ^{-\beta / n} k}=1<\frac{1}{(2 \sigma)^{n-1}} .
$$

So, we have $|D f| \in L^{1}\left([0,1]^{n}\right)$ and therefore $f \in W^{1,1}\left([0,1]^{n} ; \mathbf{R}^{n}\right)$.

The Jacobian of $f$ is locally integrable as a Jacobian of a Sobolev homeomorphism [17, Lemma 5.3 and Proposition 4.1].

Finally, let us examine the sub-exponential integrability of the distortion function of $f$. The Jacobian of $f$ is given by

$$
J_{f_{k}}(x)=a_{k}\left(a_{k}+\frac{b_{k}}{\left|x-q_{k, j}\right|_{\infty}}\right)^{n-1}
$$

at almost every $x \in A_{k, j}$. Thus, $K_{f}$ is bounded by

$$
K_{f_{k}}(x) \leq C_{0}^{n}\left(1+\frac{b_{k}}{a_{k}\left|x-q_{k, j}\right|_{\infty}}\right) \leq C_{0}^{n} \frac{1-2 \sigma}{2 \sigma} \frac{1}{\left(\frac{\log k}{\log (k-1)}\right)^{\beta / n}-1}=: C_{0}^{n} K_{k}
$$

for almost every $x \in A_{k, j}$, when $k \geq k_{0}$. This gives the estimate for $p>0$

$$
\int_{[0,1]^{n}} \exp \left(\frac{p K_{f}}{1+\log K_{f}}\right) \leq C+\sum_{k=k_{0}}^{\infty}(2 \sigma)^{n(k-1)} \exp \left(\frac{p C_{0}^{n} K_{k}}{1+\log K_{k}}\right)
$$

with a constant $C=C(n, \sigma, \beta)>0$. By Lemma 1 below,

$$
\lim _{k \rightarrow \infty} \frac{\exp \left(\frac{p C_{0}^{n} K_{k+1}}{1+\log K_{k+1}}\right)}{\exp \left(\frac{p C_{0}^{n} K_{k}}{1+\log K_{k}}\right)}=\exp \left(p C_{0}^{n} \frac{1-2 \sigma}{2 \sigma} \frac{n}{\beta}\right)
$$


and thus, by the Ratio Test, the series above converges provided

$$
\exp \left(p C_{0}^{n} \frac{1-2 \sigma}{2 \sigma} \frac{n}{\beta}\right)<(2 \sigma)^{-n}
$$

So, we have

$$
e^{\frac{K_{f}}{1+\log K_{f}}} \in L_{\operatorname{loc}}^{p}(\Omega)
$$

for all $p<p_{0}=\frac{\beta}{C_{0}^{n}} \frac{2 \sigma}{1-2 \sigma} \log \frac{1}{2 \sigma}$. Choosing $\sigma$ close enough to $1 / 2$, we can make $p_{0}$ as close to $\beta / C_{0}^{n}$ as we wish.

The following lemma verifies (3).

Lemma 1. We have

$$
\lim _{k \rightarrow \infty} \frac{\exp \left(\frac{p C_{0}^{n} K_{k+1}}{1+\log K_{k+1}}\right)}{\exp \left(\frac{p C_{0}^{n} K_{k}}{1+\log K_{k}}\right)}=\exp \left(p C_{0}^{n} \frac{1-2 \sigma}{2 \sigma} \frac{n}{\beta}\right),
$$

where $K_{k}$ is as defined in (2).

Proof. Straightforward calculations give us

$$
\begin{aligned}
& \frac{p C_{0}^{n} K_{k+1}}{1+\log K_{k+1}}-\frac{p C_{0}^{n} K_{k}}{1+\log K_{k}} \\
& =p C_{0}^{n} \alpha \frac{\left(\frac{1}{T_{k+1}}-\frac{1}{T_{k}}\right) \log ^{-1} \frac{\alpha}{T_{k+1}} \log ^{-1} \frac{\alpha}{T_{k}}+\frac{1}{T_{k+1}} \log ^{-1} \frac{\alpha}{T_{k+1}}-\frac{1}{T_{k}} \log ^{-1} \frac{\alpha}{T_{k}}}{1+\log ^{-1} \frac{\alpha}{T_{k+1}} \log ^{-1} \frac{\alpha}{T_{k}}+\log ^{-1} \frac{\alpha}{T_{k+1}}+\log ^{-1} \frac{\alpha}{T_{k}}}
\end{aligned}
$$

where $\alpha=(1-2 \sigma) /(2 \sigma)$ and $T_{t}=(\log t / \log (t-1))^{\beta / n}-1$ for $t \in[3, \infty[$. Notice that $T_{t} \rightarrow 0$ as $t \rightarrow \infty$. Thus, in order to prove this lemma, it is enough to show that the numerator of the fraction above goes to $n / \beta$ as $k$ tends to infinity. We demonstrate it by the following two observations:

$$
\lim _{k \rightarrow \infty}\left(\frac{1}{T_{k+1}}-\frac{1}{T_{k}}\right) \log ^{-1} \frac{\alpha}{T_{k+1}} \log ^{-1} \frac{\alpha}{T_{k}}=0
$$

and

$$
\lim _{k \rightarrow \infty}\left(\frac{1}{T_{k+1}} \log ^{-1} \frac{\alpha}{T_{k+1}}-\frac{1}{T_{k}} \log ^{-1} \frac{\alpha}{T_{k}}\right)=\frac{n}{\beta} .
$$

The main tool here is the mean-value theorem. Let us first examine the difference $\frac{1}{T_{k+1}}-\frac{1}{T_{k}}$. There exists a sequence $\left\{\zeta_{k}\right\}_{k=3}^{\infty}$ of numbers between 0 and 1 such that

$$
\frac{1}{T_{k+1}}-\frac{1}{T_{k}}=u(k+1)-u(k)=u^{\prime}\left(k+\zeta_{k}\right)
$$

where

$$
u(t)=\frac{\log ^{\beta / n}(t-1)}{\log ^{\beta / n} t-\log ^{\beta / n}(t-1)} .
$$

We have

$$
u^{\prime}(t)=\frac{\beta}{n} \frac{\left(\frac{1}{t-1} \log ^{-1}(t-1)-\frac{1}{t} \log ^{-1} t\right) \log ^{\beta / n}(t-1) \log ^{\beta / n} t}{\left(\log ^{\beta / n} t-\log ^{\beta / n}(t-1)\right)^{2}} .
$$


We apply the mean-value theorem again in order to replace the differences both in the numerator and in the denominator with multiplicative terms. We obtain for $t>3$

$$
\begin{aligned}
u^{\prime}(t) & =\frac{n}{\beta} \frac{\left(t-\theta_{t}\right)^{2}}{\left(t-\eta_{t}\right)^{2}} \frac{\left(\log \left(t-\eta_{t}\right)+1\right) \log ^{\beta / n}(t-1) \log ^{\beta / n} t}{\log ^{2 \beta / n-2}\left(t-\theta_{t}\right) \log ^{2}\left(t-\eta_{t}\right)} \\
& <\frac{n}{\beta} \frac{t^{2}}{(t-1)^{2}} \frac{(\log t+1) \log ^{2 \beta / n+2} t}{\log ^{2 \beta / n+2}(t-1)}<\frac{9 n \cdot 2^{2 \beta / n}}{\beta}(\log t+1),
\end{aligned}
$$

where $\left.\eta_{t}, \theta_{t} \in\right] 0,1[$.

Next, let us observe that

$$
\begin{aligned}
\frac{1}{T_{t}} & =\frac{\log ^{\beta / n}(t-1)}{\log ^{\beta / n} t-\log ^{\beta / n}(t-1)}=\frac{n}{\beta} \frac{\left(t-\delta_{t}\right) \log ^{\beta / n}(t-1)}{\log ^{\beta / n-1}\left(t-\delta_{t}\right)} \\
& =\frac{n}{\beta}\left(t-\delta_{t}\right) M_{t} \log \left(t-\delta_{t}\right),
\end{aligned}
$$

where $\left.\delta_{t} \in\right] 0,1\left[\right.$ and $M_{t}=\left(\log (t-1) / \log \left(t-\delta_{t}\right)\right)^{\beta / n} \rightarrow 1$ as $t \rightarrow \infty$. Finally, we obtain for large $k$

$$
\begin{aligned}
0 & <\left(\frac{1}{T_{k+1}}-\frac{1}{T_{k}}\right) \log ^{-1} \frac{\alpha}{T_{k+1}} \log ^{-1} \frac{\alpha}{T_{k}} \\
& <\frac{9 n \cdot 2^{2 \beta / n}}{\beta} \frac{\log (k+1)+1}{\left(\log (k-1)+\log \left(\frac{n \alpha}{\beta} M_{k} \log (k-1)\right)\left(\log k+\log \left(\frac{n \alpha}{\beta} M_{k+1} \log k\right)\right)\right.} \\
& <\frac{9 n \cdot 2^{2 \beta / n}}{\beta} \frac{\log (k+1)+1}{\log ^{2}(k-1)} \rightarrow 0
\end{aligned}
$$

as $k \rightarrow \infty$.

It remains to examine the difference

$$
\frac{1}{T_{k+1}} \log ^{-1} \frac{\alpha}{T_{k+1}}-\frac{1}{T_{k}} \log ^{-1} \frac{\alpha}{T_{k}}=v(k+1)-v(k),
$$

where $v(t)=\frac{1}{T_{t}} \log ^{-1} \frac{\alpha}{T_{t}}$. Obviously, it is enough to prove that $\lim _{t \rightarrow \infty} v^{\prime}(t)=n / \beta$. Let us calculate

$$
\begin{aligned}
v^{\prime}(t) & =\frac{\beta}{n} \frac{\log ^{\beta / n-1} t}{\log ^{\beta / n+1}(t-1)} \frac{t \log t-(t-1) \log (t-1)}{t(t-1)} \frac{1-\log ^{-1} \frac{\alpha}{T_{t}}}{T_{t}^{2} \log \frac{\alpha}{T_{t}}} \\
& =\frac{\beta}{n} \frac{\log ^{\beta / n-1} t}{\log ^{\beta / n+1}(t-1)} \frac{\left(\log \left(t-\kappa_{t}\right)+1\right)}{t(t-1)} \frac{1-\log ^{-1} \frac{\alpha}{T_{t}}}{T_{t}^{2} \log \frac{\alpha}{T_{t}}} \\
& =\frac{\beta}{n} N_{t} \frac{1-\log ^{-1} \frac{\alpha}{T_{t}}}{t(t-1) T_{t}^{2} \log (t-1) \log \frac{\alpha}{T_{t}}},
\end{aligned}
$$

where $\left.\kappa_{t} \in\right] 0,1\left[\right.$ and $N_{t} \rightarrow 1$ as $t \rightarrow \infty$. We use the representation (4) again to obtain

$$
v^{\prime}(t)=\frac{n}{\beta} \frac{\left(t-\delta_{t}\right)^{2}}{t(t-1)} \frac{N_{t} M_{t}^{2}\left(1-\log ^{-1} \frac{\alpha}{T_{t}}\right) \log ^{2}\left(t-\delta_{t}\right)}{\left(\log \left(t-\delta_{t}\right)+\log \left(\frac{n \alpha}{\beta} M_{t} \log \left(t-\delta_{t}\right)\right)\right) \log (t-1)} \rightarrow \frac{n}{\beta}
$$

as $t \rightarrow \infty$. 


\section{Proof of Theorem 1}

Without loss of generality, we may assume for the rest of the paper that $\Omega$ is connected. Moreover, using the $\sigma$-additivity of the generalized Hausdorff measure, we may assume in what follows, that $\Omega$ is bounded and $e^{\frac{p K_{f}}{1+\log K_{f}}}$ is globally integrable in $\Omega$. We will use a higher integrability result for the Jacobian from [5] to establish the desired dimension distortion estimate.

Proof of Theorem 1. Corollary 3.3 from [5] gives us a constant $c=c(n)>0$ such that $|D f| \in L_{\mathrm{loc}}^{P_{\beta}}(\Omega)$ and $J_{f} \log ^{\beta} \log \left(e^{e}+J_{f}\right) \in L_{\mathrm{loc}}^{1}(\Omega)$ for all $\beta<c p$, where

$$
P_{\beta}(t)=\frac{t^{n}}{\log (e+t) \log ^{1-\beta}\left(\log \left(e^{e}+t\right)\right)}
$$

Fix some $q \in] n-1, n[$. The integrability of the differential of $f$ guarantees that $f \in W_{\text {loc }}^{1, q}(\Omega)$. In order to conclude $f^{-1} \in W_{\text {loc }}^{1, q}(f(\Omega))$ by [11, Theorem 4.2], we also need $K_{f}^{\frac{(q-1) q}{2 q-n}}$ to be integrable in $\Omega$, which is clearly true as $K_{f}$ is sub-exponentially integrable. Finally, the regularity of the weak derivatives of $f$ is enough to guarantee Det $D f=J_{f}$, since the function $P_{\beta}$ satisfies the assumptions (i) and (ii) of Theorem 1.2 in [20]. The desired equality Det $D f=J_{f}$ follows also from the remark in $[10$, p. 594]. All this makes the application of Lemma 2 possible, concluding the proof of the theorem.

Lemma 2. Let $f \in W_{\text {loc }}^{1, q}\left(\Omega ; \mathbf{R}^{n}\right), \Omega \subset \mathbf{R}^{n}(n \geq 2$ and $q>n-1)$, be a homeomorphism, such that Det $D f=J_{f}, J_{f}(x) \geq 0$ for almost every $x \in \Omega$ and $J_{f} \log ^{\beta} \log \left(e^{e}+J_{f}\right) \in L_{\mathrm{loc}}^{1}(\Omega)$ for some $\beta$. If $n>2$, assume in addition that $f^{-1} \in$ $W_{\text {loc }}^{1, q}\left(\Omega ; \mathbf{R}^{n}\right)$. Then $\mathscr{H}^{h_{n, \beta}}(f(E))=0$, whenever $E \subset \Omega$ is such that $\operatorname{dim}_{\mathscr{H}} E<n$.

The assumptions $f \in W_{\text {loc }}^{1, q}\left(\Omega ; \mathbf{R}^{n}\right)$ and Det $D f=J_{f}$ are due to our intention to use Lemma 3.2 from [14]. Before proving Lemma 2, let us state the following auxillary result. This lemma is Lemma 9 from [22], its proof is a standard extension to higher dimensions of the planar case [18, Lemma 3.1].

\section{Lemma 3.}

(i) Let $f: \Omega \rightarrow f(\Omega) \subset \mathbf{R}^{n}, n>2$, be a homeomorphism such that $f^{-1} \in$ $W_{\text {loc }}^{1, q}\left(\Omega ; \mathbf{R}^{n}\right)$ for some $\left.q \in\right] n-1, n[$. Then there exists a set $F \subset f(\Omega)$ such that $\mathscr{H}^{n-\frac{q}{2}}(F)=0$ and for all $y \in f(\Omega) \backslash F$ there exist constants $C_{y}>0$ and $r_{y}>0$ such that

$$
\operatorname{diam}\left(f^{-1}(B(y, r))\right) \leq C_{y} r^{1 / 2}
$$

for all $0<r<r_{y}$.

(ii) If $n=2$, (i) is true with the assumption $f^{-1} \in W_{\text {loc }}^{1, q}\left(\Omega ; \mathbf{R}^{n}\right)$ replaced by the condition $f \in W_{\text {loc }}^{1,1}(\Omega)$ and with $q=1$, that is, with $\mathscr{H}^{3 / 2}(F)=0$ for the exceptional set $F$.

Proof of Lemma 2. The proof repeats the strategy of the proof of Theorem 1.1 from [21]. As in Lemma 3.2 from [18], using Lemma 3, we may represent the image 
set $\Omega^{\prime}=f(\Omega)$ in the following form

$$
\Omega^{\prime}=F \cup \bigcup_{j=1}^{\infty} \bigcup_{k=1}^{\infty}\left\{y \in \Omega^{\prime}: \operatorname{diam}\left(f^{-1}(B(y, r))\right) \leq k r^{\frac{1}{2}} \text { for all } r \in\right] 0,1 / j[\},
$$

obtaining a decomposition $\Omega^{\prime}=\bigcup_{i=0}^{\infty} F_{i}$ and a collection of constants $\left\{C_{i}\right\}_{i=1}^{\infty},\left\{R_{i}\right\}_{i=1}^{\infty}$, such that $\mathscr{H}^{h_{n, \beta}}\left(F_{0}\right)=0$ and for each $i=1,2, \ldots$, we have $1 \leq C_{i}<\infty, R_{i}>0$ and

$$
f^{-1}\left(\left(f(A) \cap F_{i}\right)+\left(\frac{r}{C_{i}}\right)^{2}\right) \subset A+r
$$

for every $A \subset \Omega$ and for every $r \in] 0, R_{i}[$.

Fix $i \geq 1$. Let us show that $\mathscr{H}^{h_{n, \beta}}\left(f(E) \cap F_{i}\right)=0$. Take some

$$
s \in] \max \left\{\operatorname{dim}_{\mathscr{H}} E, n-1\right\}, n[
$$

and put $\sigma=\frac{n-s}{2}<\frac{1}{2}$. Choose $\left.r_{0} \in\right] 0, e^{-1 / \sigma^{2}}\left[\right.$ small enough to guarantee $\log ^{\beta}\left(2 \log \frac{C_{i}}{r}\right)$ $\leq r^{-\sigma}$ for all $\left.\left.r \in\right] 0, r_{0}\right]$.

Fix now $\varepsilon>0$. Using the absolute continuity of the Lebesgue integral and the given integrability of the Jacobian, we may find a number $\delta>0$, such that

$$
\int_{A} J_{f}(x) \log ^{\beta} \log \left(e^{e}+J_{f}(x)\right) d x<\varepsilon
$$

for each $A \subset \Omega$ such that $\mathscr{L}^{n}(A)<\delta$.

Since $\mathscr{H}^{s}(E)=0$, we may find a countable collection of balls $\left\{B\left(x_{j}, r_{j}\right)\right\}_{j=1}^{\infty}$ covering $E$ and having radii less than $\min \left\{r_{0}, R_{i}, \frac{1}{C_{i}}\right\}$, such that

$$
\sum_{j=1}^{\infty} 2^{n} \omega_{n} r_{j}^{s}<\min \{\varepsilon, \delta\}
$$

Now, write $F_{i, j}=F_{i} \cap f\left(B\left(x_{j}, r_{j}\right)\right)$ for each $j \in \mathbf{N}$. Notice by $(6)$ that $f^{-1}\left(F_{i, j}+\right.$ $\left.R_{i, j}\right) \subset B\left(x_{j}, 2 r_{j}\right)$, where $R_{i, j}=\left(\frac{r_{j}}{C_{i}}\right)^{2}$.

Next, we use the $5 r$-covering theorem to find an at most countable subcollection of pairwise disjoint balls $\left\{B\left(y_{k}, \rho_{k}\right)\right\}_{k \in K}$ from the collection

$$
\bigcup_{j=1}^{\infty}\left\{B\left(y, R_{i, j}\right): y \in F_{i, j}\right\}
$$

so that

$$
F_{i} \cap f(E) \subset \bigcup_{k \in K} B\left(y_{k}, 5 \rho_{k}\right),
$$

where, for each $k \in K$, we have $y_{k} \in F_{i, j}$ for some $j=j(k)$ and $\rho_{k}=R_{i, j(k)}$.

Since $r_{j}<e^{-1 / \sigma^{2}}<e^{-4}$ for all $j \in \mathbf{N}$, we have $\frac{1}{10 R_{i, j(k)}}>\frac{C_{i}^{2} e^{8}}{10}>e$ for $k \in K$. Lemma 3.2 from [14] yields

$$
\mathscr{L}^{n}\left(B\left(y_{k}, R_{i, j(k)}\right)\right) \leq \int_{f^{-1}\left(B\left(y_{k}, R_{i, j(k)}\right)\right)} J_{f}(x) d x
$$

for all $k \in K$. Thus, we may estimate 


$$
\begin{aligned}
& \mathscr{H}_{10 r_{0}}^{h_{n, \beta}}\left(F_{i} \cap f(E)\right) \leq \sum_{k \in K} 10^{n} R_{i, j(k)}^{n} \log ^{\beta} \log \left(\frac{1}{10 R_{i, j(k)}}\right) \\
& \leq \frac{10^{n}}{\omega_{n}} \sum_{k \in K} \mathscr{L}^{n}\left(B\left(y_{k}, R_{i, j(k)}\right)\right) \log ^{\beta} \log \left(\frac{1}{R_{i, j(k)}}\right) \\
& \leq \frac{10^{n}}{\omega_{n}} \sum_{k \in K} \int_{f^{-1}\left(B\left(y_{k}, R_{i, j(k)}\right)\right)} \log ^{\beta} \log \left(\frac{1}{R_{i, j(k)}}\right) J_{f}(x) d x \\
&= \frac{10^{n}}{\omega_{n}} \sum_{k \in K}\left(\int_{\left\{x \in f^{-1}\left(B\left(y_{k}, R_{i, j(k)}\right)\right): J_{f}(x)<r_{j(k)}^{-\sigma}\right\}} \log ^{\beta} \log \left(\frac{1}{R_{i, j(k)}}\right) J_{f}(x) d x\right. \\
&\left.+\int_{\left\{x \in f^{-1}\left(B\left(y_{k}, R_{i, j(k)}\right)\right): J_{f}(x) \geq r_{j(k)}^{-\sigma}\right\}} \log ^{\beta} \log \left(\frac{1}{R_{i, j(k)}}\right) J_{f}(x) d x\right) \\
& \leq \frac{10^{n}}{\omega_{n}} \sum_{k \in K} r_{j(k)}^{-2 \sigma} \mathscr{L}^{n}\left(f^{-1}\left(B\left(y_{k}, R_{i, j(k)}\right)\right)\right) \\
&+\frac{10^{n}}{\omega_{n}} \sum_{k \in K} \frac{\log ^{\beta} \log \left(1 / R_{i, j(k)}\right)}{\log ^{\beta} \log \left(e^{e}+1 / r_{j(k)}^{\sigma}\right)} \int_{f^{-1}\left(B\left(y_{k}, R_{i, j(k)}\right)\right)} J_{f} \log ^{\beta} \log \left(e^{e}+J_{f}\right)
\end{aligned}
$$

using the fact that $\log ^{\beta}\left(2 \log \frac{C_{i}}{r_{j}}\right) \leq r_{j}^{-\sigma}$ for all $j \in \mathbf{N}$. Let us estimate the first term in the last sum. By grouping the balls according to $j(k)$ and using the relation $f^{-1}\left(F_{i, j}+R_{i, j}\right) \subset B\left(x_{j}, 2 r_{j}\right)$, we get

$$
\begin{aligned}
\sum_{k \in K} r_{j(k)}^{-2 \sigma} \mathscr{L}^{n}\left(f^{-1}\left(B\left(y_{k}, R_{i, j(k)}\right)\right)\right) & =\sum_{j=1}^{\infty} r_{j}^{s-n} \sum_{\substack{k \in K \\
j(k)=j}} \mathscr{L}^{n}\left(f^{-1}\left(B\left(y_{k}, R_{i, j}\right)\right)\right) \\
& \leq \sum_{j=1}^{\infty} r_{j}^{s-n} \mathscr{L}^{n}\left(B\left(x_{j}, 2 r_{j}\right)\right)=\sum_{j=1}^{\infty} 2^{n} \omega_{n} r_{j}^{s}<\varepsilon .
\end{aligned}
$$

Let us now estimate the second term in the sum. Since $r_{j}<\frac{1}{C_{i}}$ and $r_{j}<e^{-1 / \sigma^{2}}<e^{-4}$ for all $j \in \mathbf{N}$, we obtain for each $k \in K$

$$
\begin{aligned}
\frac{\log ^{\beta} \log \left(1 / R_{i, j(k)}\right)}{\log ^{\beta} \log \left(e^{e}+1 / r_{j(k)}^{\sigma}\right)} & \leq \frac{\log ^{\beta}\left(2 \log \frac{C_{i}}{r_{j(k)}}\right)}{\log ^{\beta}\left(\sigma \log \frac{1}{r_{j(k)}}\right)} \leq \frac{\log ^{\beta}\left(4 \log \frac{1}{r_{j(k)}}\right)}{\log ^{\beta}\left(\sigma \log \frac{1}{r_{j(k)}}\right)} \\
& =\left(\frac{\log 4+\log \log \frac{1}{r_{j(k)}}}{\log \sigma+\log \log \frac{1}{r_{j(k)}}}\right)^{\beta} \leq 2^{2 \beta} .
\end{aligned}
$$

Using the pairwise disjointness of $f^{-1}\left(B\left(y_{k}, R_{i, j(k)}\right)\right), k \in K$, and the fact that $f^{-1}\left(F_{i, j}+R_{i, j}\right) \subset B\left(x_{j}, 2 r_{j}\right)$ for all $j \in \mathbf{N}$, we conclude

$$
\begin{aligned}
& \sum_{k \in K} \frac{\log ^{\beta} \log \left(1 / R_{i, j(k)}\right)}{\log ^{\beta} \log \left(e^{e}+1 / r_{j(k)}^{\sigma}\right)} \int_{f^{-1}\left(B\left(y_{k}, R_{i, j(k)}\right)\right)} J_{f} \log ^{\beta} \log \left(e^{e}+J_{f}\right) \\
& \leq 2^{2 \beta} \sum_{k \in K} \int_{f^{-1}\left(B\left(y_{k}, R_{i, j(k)}\right)\right)} J_{f} \log ^{\beta} \log \left(e^{e}+J_{f}\right)
\end{aligned}
$$




$$
\begin{aligned}
& \leq 2^{2 \beta} \int_{\bigcup_{k \in K} f^{-1}\left(B\left(y_{k}, R_{i, j(k)}\right)\right)} J_{f} \log ^{\beta} \log \left(e^{e}+J_{f}\right) \\
& \leq 2^{2 \beta} \int_{\bigcup_{j=1}^{\infty} B\left(x_{j}, 2 r_{j}\right)} J_{f} \log ^{\beta} \log \left(e^{e}+J_{f}\right) \leq 2^{2 \beta} \varepsilon,
\end{aligned}
$$

since

$$
\mathscr{L}^{n}\left(\bigcup_{j=1}^{\infty} B\left(x_{j}, 2 r_{j}\right)\right) \leq \sum_{j=1}^{\infty} 2^{n} \omega_{n} r_{j}^{n} \leq \sum_{j=1}^{\infty} 2^{n} \omega_{n} r_{j}^{s}<\delta .
$$

\section{Planar case}

As it was mentioned in the first section, the assumption on $f$ to be a homeomorphism can be avoided in the plane due to factorization of the solutions of the Beltrami equation. The Beltrami equation is an equation in the complex plane $\mathbf{C}$ of the form

$$
\bar{\partial} f(z)=\mu(z) \partial f(z)
$$

where $\bar{\partial}=\frac{1}{2}\left(\partial_{x}+i \partial_{y}\right)$ and $\partial=\frac{1}{2}\left(\partial_{x}-i \partial_{y}\right)$. The function $\mu$ is the Beltrami coefficient of the mapping $f$ (provided $f$ is a solution of (7) in some sense). Given an abstract Beltrami coefficient $\mu(z)$, such that $|\mu(z)|<1$ almost everywhere, we can associate to $\mu$ a real-valued function $K=\frac{1+|\mu|}{1-|\mu|}$, called a distortion function of the Beltrami equation. The terminology is natural, as the Beltrami equation yields the distortion inequality

$$
|D f(z)|^{2} \leq K(z) J_{f}(z)
$$

for its $W_{\text {loc }}^{1,1}$-solutions. Conversely, a mapping $f$ with finite optimal distortion function $K_{f}(z)$ satisfies almost everywhere the Beltrami equation with the associated Beltrami coefficient $\mu_{f}(z)=\bar{\partial} f(z) / \partial f(z)$, when $\partial f(z) \neq 0\left(\mu_{f}(z)=0\right.$ otherwise). In this case, the distortion function of this Beltrami equation equals $K_{f}$ and $|\mu(z)|=\frac{K_{f}(z)-1}{K_{f}(z)+1}<1$ for almost every $z$.

Proof of Theorem 2. Let $\mathscr{A}$ be defined by $\mathscr{A}(t)=p \frac{t}{1+\log t}-p$. Thus, our subexponential integrability assumption on $f$ may be rewritten as $e^{\mathscr{A}\left(K_{f}(z)\right)} \in L^{1}(\Omega)$. Clearly, the function $\mathscr{A}$ satisfies conditions 1-3 from [2, pp. 570-571], so, we may apply Theorem 20.5.2 in [2], which gives the unique principal solution $g$ to the global Beltrami equation that is satisfied by $f$ almost everywhere in $\Omega$. See $[2$, Definition 20.0.4] for the definition of the principal solution of the Beltrami equation. In particular, $g$ is homeomorphic. In addition, Theorem 20.5.2 in [2] asserts that $f$ can be factorized as $f=\phi \circ g$ (where $\phi$ is holomorphic in $g(\Omega)$ ), provided $f \in W_{\text {loc }}^{1, P}(\Omega)$ for

$$
P(t)= \begin{cases}t^{2}, & 0 \leq t \leq 1, \\ \frac{t^{2}}{\mathscr{A}^{-1}\left(\log t^{2}\right)}, & t \geq 1,\end{cases}
$$

which is true by [2, Theorem 20.5.1]. 
Higher integrability of the Jacobian for $g$ follows from Theorem 1 in [8], yielding $J_{g} \log ^{\beta} \log \left(e^{e}+J_{g}\right) \in L_{\mathrm{loc}}^{1}(\Omega)$ and

$$
\frac{|D f|^{2}}{\log (e+|D f|) \log ^{1-\beta} \log \left(e^{e}+|D f|\right)} \in L_{\mathrm{loc}}^{1}(\Omega)
$$

for all $\beta<p$. This allows to use Lemma 2, giving $\mathscr{H}^{h_{2, \beta}}(g(E))=0$ for all $\beta<p$ and each set $E \subset \Omega$ such that $\operatorname{dim}_{\mathscr{H}} E<2$. Finally, as $\phi$ is locally Lipschitz, we obtain $\mathscr{H}^{h_{2, \beta}}(f(E))=0$ for such $\beta$ and $E$.

Acknowledgments. The authors thank Pekka Koskela for suggesting this problem and the referee for his/her careful comments.

\section{References}

[1] Astala, K., J. T. Gill, S. Rohde, and E. Saksman: Optimal regularity for planar mappings of finite distortion. - Ann. Inst. H. Poincaré Anal. Non Linéaire 27:1, 2010, 1-19.

[2] Astala, K., T. Inaniec, and G. Martin: Elliptic partial differential equations and quasiconformal mappings in the plane. - Princeton Mathematical Series 48, Princeton Univ. Press, Princeton, NJ, 2009.

[3] BALL, J. M.: Convexity conditions and existence theorems in nonlinear elasticity. - Arch. Rational Mech. Anal. 63:4, 1976/77, 337-403.

[4] Bojarski, B. V.: Homeomorphic solutions of Beltrami systems. - Dokl. Akad. Nauk SSSR (N.S.), 102, 1955, 661-664.

[5] Clop, A., and P. Koskela: Orlicz-Sobolev regularity of mappings with subexponentially integrable distortion. - Atti Accad. Naz. Lincei Cl. Sci. Fis. Mat. Natur. Rend. Lincei (9) Mat. Appl. 20:4, 2009, 301-326.

[6] Gehring, F. W.: The $L^{p}$-integrability of the partial derivatives of a quasiconformal mapping. - Acta Math. 130, 1973, 265-277.

[7] Gehring, F. W., and J. VÄISÄLÄ: Hausdorff dimension and quasiconformal mappings. - J. London Math. Soc. (2) 6, 1973, 504-512.

[8] Gill, J. T.: Planar maps of sub-exponential distortion. - Ann. Acad. Sci. Fenn. Math. 35:1, 2010, 197-207.

[9] Greco, L.: A remark on the equality $\operatorname{det} D f=\operatorname{Det} D f$. - Differential Integral Equations, 6:5, 1993, 1089-1100.

[10] Greco, L.: Sharp integrability of nonnegative Jacobians. - Rend. Mat. Appl. (7) 18:3, 1998, $585-600$.

[11] Hencl, S., P. Koskela, and J. Malý: Regularity of the inverse of a Sobolev homeomorphism in space. - Proc. Roy. Soc. Edinburgh Sect. A 136:6, 2006, 1267-1285.

[12] Herron, D. A., and P. Koskela: Mappings of finite distortion: gauge dimension of generalized quasicircles. - Illinois J. Math. 47:4, 2003, 1243-1259.

[13] Iwaniec, T., and C. Sbordone: On the integrability of the Jacobian under minimal hypotheses. - Arch. Rational Mech. Anal. 119:2, 1992, 129-143.

[14] Kauhanen, J., P. Koskela, and J. MalÝ: Mappings of finite distortion: condition N. Michigan Math. J. 49:1, 2001, 169-181.

[15] Kauhanen, J., P. Koskela, and J. MalÝ: Mappings of finite distortion: discreteness and openness. - Arch. Ration. Mech. Anal. 160:2, 2001, 135-151.

[16] Kauhanen, J., P. Koskela, J. Malý, J. Onninen, and X. Zhong: Mappings of finite distortion: sharp Orlicz-conditions. - Rev. Mat. Iberoamericana 19:3, 2003, 857-872. 
[17] Koskela, P.: Lectures on quasiconformal and quasisymmetric mappings. - http://users.jyu.fi/ $\sim$ pkoskela/quasifinal.pdf.

[18] Koskela, P., A. Zapadinskaya, and T. ZÜrcher: Generalized dimension distortion under planar Sobolev homeomorphisms. - Proc. Amer. Math. Soc. 137:11, 2009, 3815-3821.

[19] Koskela, P., A. Zapadinskaya, and T. ZÜrcher: Mappings of finite distortion: generalized Hausdorff dimension distortion. - J. Geom. Anal. 20:3, 2010, 690-704.

[20] Koskela, P., and X. Zhong: Minimal assumptions for the integrability of the Jacobian. Ricerche Mat. 51:2, 2002, 297-311.

[21] Rajala, T.: Planar Sobolev homeomorphisms and Hausdorff dimension distortion. - Proc. Amer. Math. Soc. 139:5, 2011, 1825-1829.

[22] Rajala, T., A. Zapadinskaya, and T. ZÜrcher: Generalized Hausdorff dimension distortion in Euclidean spaces under Sobolev mappings. - Preprint, http://arxiv.org/abs/1007.2091.

Received 17 June 2010

Revised received 27 May 2011 